\title{
Research on Unbalance Phase Current of 500kV AC Transmission Line in Yibin UHV Converter Station
}

\author{
M. TANG, C. ZHANG, M. Li, L.J. Ding, H. Zhang, W. Wei \& F. Tang \\ Electric power science research institute of state grid Sichuan electric power company, Chengdu, \\ 610071
}

KEYWORD: Parallel transmission line; Unbalanced Current; Inductor Model

ABSTRACT: Unbalance phase current appeared in the $500 \mathrm{kV}$ overhead transmission line during the system commission of $\pm 800 \mathrm{kV}$ Binjin UHV DC transmission project. An inductor model of parallel lines on same tower was established for theoretical analysis. Electromagnetic transient model was also established in EMTDC/PSCAD. Both theoretical analysis and simulation result pointed that: the inappropriate overhead line arrangement of tower induces the unbalance phase current. The mechanism of unbalance phase current was revealed and optimal arrangement of parallel transmission line on same tower was given.

\section{INTRODUCTION}

$\pm 800 \mathrm{kV}$ Binjin ultra-high voltage (UHV) DC transmission project, rated transmission capacity $8000 \mathrm{MW}$, has the maximum capacity in the world so far. As another important transmission line for the hydropower of Sichuan, it relaxes intensity status of power lag in east china area.

During the system commission, obviously unbalance phase current was found in the two $500 \mathrm{kV}$ $\mathrm{AC}$ transmission lines between Yibin converter station and Fulong converter station. The unbalance phase current will cause bad influence on primary equipment such as: motors, transformers and etc. It will also lead to incorrect operation of relay protection devices.

So, it is necessary to analyze the mechanism of unbalance phase current to decrease its influence and try to find a better solution.

\section{Unbalance Phase Current in Yibin Converter Station}

The AC switching yard of Yibin converter station adopts $3 / 2$ connection with 7 complete strings and 3 incomplete strings. Figure 1 shows the detailed connection. Unbalance phase current appeared on the two strings where Binfu I line and Binfu II line connected. The total line current also exhibited a higher unbalance degree. Table 1 shows the unbalanced 3-phase current of Binfu I line and Binfu II line in different cases.

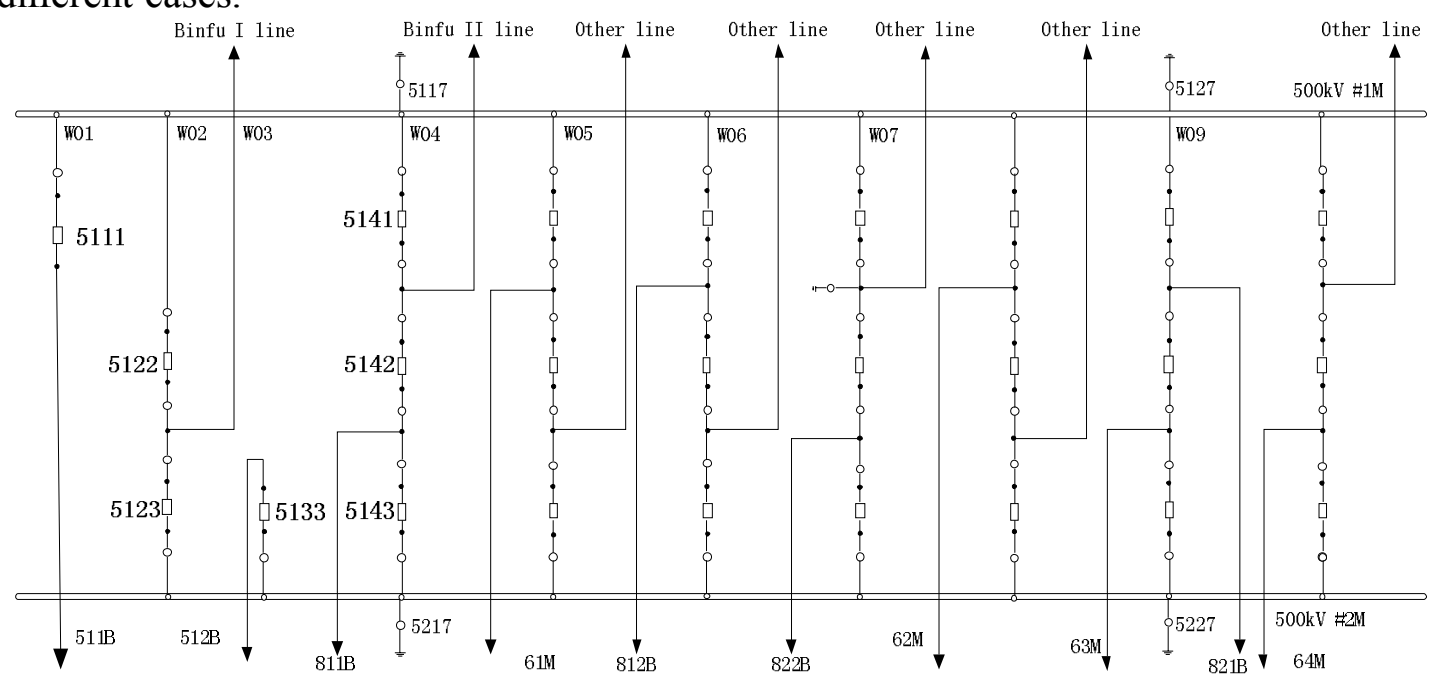

Figure $1500 \mathrm{kV}$ AC yard connect diagram of Yibin converter station 
Table 1 Unbalance 3-phase current of each line in different cases

\begin{tabular}{|c|c|c|c|c|c|c|c|}
\hline \multirow{2}{*}{$\begin{array}{c}\text { Data } \\
\text { group }\end{array}$} & \multirow{2}{*}{ Variables } & \multicolumn{3}{|c|}{ Binfu I line } & \multicolumn{3}{c|}{ Binfu II line } \\
\cline { 3 - 8 } & & A & B & C & A & B & C \\
\hline \multirow{2}{*}{1} & current (A) & 519.1 & 479.9 & 527.7 & 500.9 & 535.1 & 488.3 \\
\cline { 2 - 8 } & voltage (kV) & 528.4 & 528.6 & 528.5 & 529.4 & 529.4 & 528.2 \\
\hline \multirow{2}{*}{2} & current (A) & $\begin{array}{c}1049 . \\
8\end{array}$ & 972.6 & $\begin{array}{c}1067 . \\
2\end{array}$ & 1011.9 & 1087.3 & 988.8 \\
\cline { 2 - 8 } & voltage (kV) & 526.4 & 526.7 & 526.6 & 526.9 & 527.3 & 526.8 \\
\hline \multirow{2}{*}{3} & current (A) & $\begin{array}{c}1332 . \\
0\end{array}$ & 1235.1 & $\begin{array}{c}1356 . \\
2\end{array}$ & 1282.6 & 1380.1 & 1255.5 \\
\cline { 2 - 8 } & voltage (kV) & 530.5 & 530.2 & 530.6 & 531.4 & 531.1 & 530.0 \\
\hline
\end{tabular}

The probable cause of unbalance phase current can be divided into 3 groups: A. unbalance 3-phase current caused by system load flow; B. the unbalance resistance of GIS switch circuit; C. the unbalance parameters of 3-phase transmission line or unbalance 3-phase load.

As shown in Table.1, $I a>I c>I b$ in Binfu I line and $I b>I a>I c$ in Binfu II line in different cases. However, the summation of Binfu I line and Binfu II line is basically balanced, meaning a balanced 3phase load. Furthermore, on-site measurement reported no obvious unbalance of GIS switch circuit resistance. So, the unbalance parameters of 3-phase transmission line turns to the only reason.

\section{Modeling of Line Current}

Binfu I line and Binfu II line connect Fulong converter station and Yibin converter station with the total length of $26.926 \mathrm{~km}$ (I line $13.4 \mathrm{~km}$, II line $13.526 \mathrm{~km}$ ). The two line corridors are parallel and non-transposition. Center distance of the two tower is about $60-80 \mathrm{~m}$. The detailed phase sequence of each line is shown in Figure 2: from left to right, ABC for Binfu II line and ACB for Binfu I line.

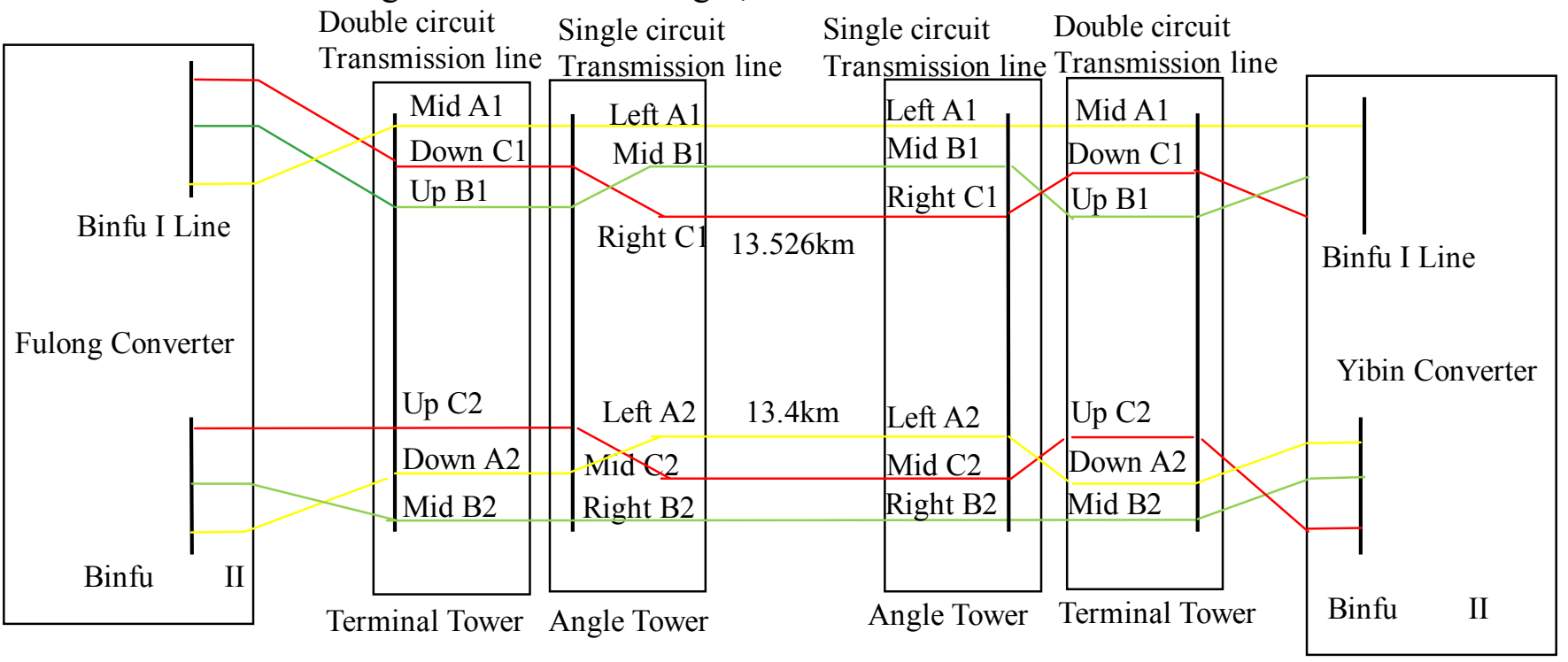

Figure 2 the current phase arrangement of Binfu I line and Binfu II line

Figure 3 shows geometrical arrangement of Binfu I line and Binfu II line.

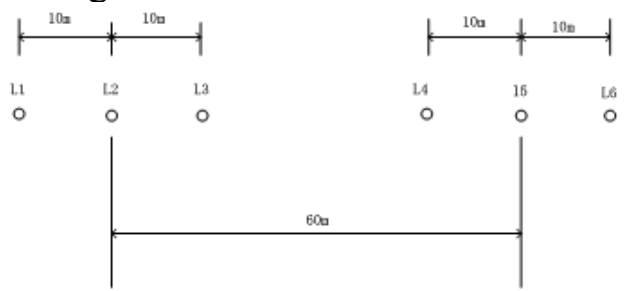

Figure 3 Geometrical models of transmission lines 
To analyze the unbalance phase current in the point of view of electric circuit, inductor model of the transmission line as well as electromagnetic equations should be established. 2-D finite element method, based on ANSYS, is used to calculate self-inductance of transmission lines and mutual inductance between them. The $6 \times 6$ inductor matrix $L$ can be obtained from the calculation result of ANSYS, where $L_{a l a l}$ means A phase self-inductance of Binfu I line, and $L_{a l b 2}$ means mutual inductance from B phase of Binfu II line to A phase of Binfu I line. The flux of each transmission line can be obtained as follows:

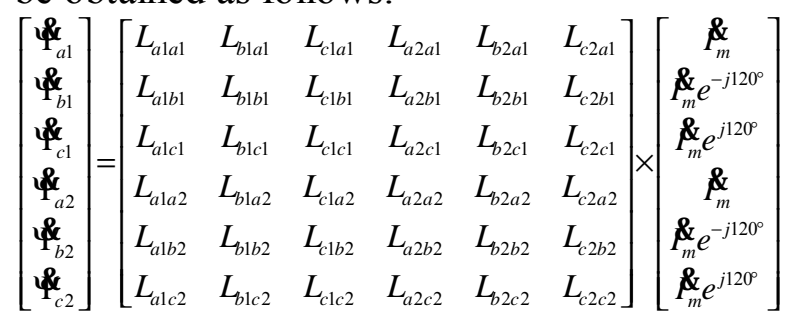

Equation 2 shows the decouple method for the flux of each transmission line.

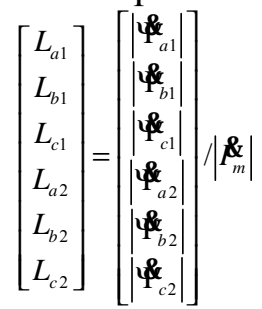

In Equation 2, $L_{a 1}, L_{b 1}, L_{c l}, L_{a 2}, L_{b 2}, L_{c 2}$ stands for the equivalent inductance for each transmission line. The resistance of each transmission line can be obtained for its specification. So, the positive sequence impedance of each transmission line can be obtained as $Z=R+j \omega L$, and the model for calculation is shown in figure 4 . The capacitance parameter is not taken into consideration in this model.

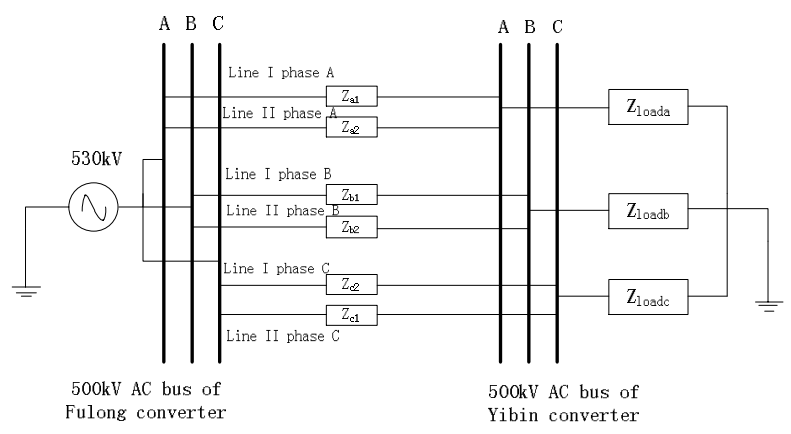

Figure 4 Calculation model for unbalance phase current

The present arrangement of transmission line is shown in Figure 3; current distribution of each line is calculated as well as other two arrangements shown in Figure 3 (a). Parameters in this calculation are: the length of Binfu I line and Binfu II line is $13.5 \mathrm{~km}$; all the lines are arranged horizontal; terminal towers and angle towers are ignored; the resistance of transmission line is $0.215 \Omega$; the total power flow is $\mathrm{P}=2400 \mathrm{MW}, \mathrm{Q}=250 \mathrm{MVar}$. 
Table.2 Calculation result under different arrangement

(a) position layout of transmission lines

\begin{tabular}{ccccccc}
\hline $\begin{array}{c}\text { Number of } \\
\text { conductors }\end{array}$ & \multicolumn{3}{c}{$\begin{array}{c}\text { Binfu I line } \\
\text { Position of conductors }\end{array}$} & C & A & Binfu II line \\
& A & B & Cosition of conductors & B & C \\
\hline 1 & L4 & L6 & L5 & L 1 & L 2 & L 3 \\
\hline 2 & L 4 & L 5 & L 6 & L 1 & L 2 & L 3 \\
\hline 3 & L 6 & L 5 & L 4 & L 1 & L 2 & L 3 \\
\hline
\end{tabular}

(b) calculation result

\begin{tabular}{c|lcc|lcc}
\hline \multirow{3}{*}{ Mode } & \multicolumn{3}{|c|}{ Calculation result of current $(\mathrm{kA})$} & \multicolumn{3}{c}{ Calculation result of impedance $(\Omega)$} \\
\cline { 2 - 7 } & Ia1 & $\mathrm{Ib} 1$ & $\mathrm{Ic} 1$ & $\mathrm{Za} 1$ & $\mathrm{Zb} 1$ & $\mathrm{Zc1}$ \\
& $\mathrm{Ia} 2$ & $\mathrm{Ib} 2$ & $\mathrm{Ic} 2$ & $\mathrm{Za} 2$ & $\mathrm{Zb} 2$ & $\mathrm{Zc2}$ \\
\hline \multirow{2}{*}{1} & 1.3101 & 1.2351 & 1.3457 & $0.2150+3.1454 \mathrm{j}$ & $0.2150+3.1815 \mathrm{j}$ & $0.2150+2.9553 \mathrm{j}$ \\
& 1.2878 & 1.3630 & 1.2523 & $0.2150+3.2003 \mathrm{j}$ & $0.2150+2.8816 \mathrm{j}$ & $0.2150+3.1769 \mathrm{j}$ \\
\hline \multirow{2}{*}{2} & 1.3137 & 1.2991 & 1.2842 & $0.2150+3.1303 \mathrm{j}$ & $0.2150+2.9071 \mathrm{j}$ & $0.2150+3.2043 \mathrm{j}$ \\
& 1.2842 & 1.2991 & 1.3137 & $0.2150+3.2024 \mathrm{j}$ & $0.2150+2.9072 \mathrm{j}$ & $0.2150+3.1319 \mathrm{j}$ \\
\hline \multirow{2}{*}{3} & 1.2990 & 1.2991 & 1.2988 & $0.2150+3.1615 \mathrm{j}$ & $0.2150+2.9074 \mathrm{j}$ & $0.2150+3.2829 \mathrm{j}$ \\
& 1.2990 & 1.2991 & 1.2989 & $0.2150+3.1614 \mathrm{j}$ & $0.2150+2.9074 \mathrm{j}$ & $0.2150+3.2825 \mathrm{j}$ \\
\hline
\end{tabular}

The calculation result shows that: inductance of $500 \mathrm{kV}$ transmission line is much larger than its resistance. When positive sequence current is injected, the inductance of side lines is basically equal to each other, but the mid line's inductance is smaller than side line.

According to Figure 2, B phase of Binfu I line was arranged in side and B phase of Binfu II line was arranged in the mid, so $\left|Z_{b 1}\right|>\left|Z_{b 2}\right|, I_{b 1}<I_{b 2},\left|Z_{a 1}\right| \approx\left|Z_{a 2}\right|, I_{a 1} \approx I_{a 2},\left|Z_{c 1}\right|<\left|Z_{c 2}\right|, I_{c 1}>I_{c 2}$, it is obvious that the theoretical calculation is highly consistent with the measurement on site.

Mode 2 and 3 adopted positive phase sequence (ABC-ABC) arrangement and inverse phase sequence arrangement respectively. There is no obvious parameter difference for the same phase in Mode 2 and 3, so the current in each transmission line can be distributed equally.

\section{Simulation for electromagnetic transient analysis}

To confirm the theoretical analysis, electromagnetic transient simulation was carried out based on EMTDC/PSCAD.

The $500 \mathrm{kV}$ transmission line model was built as shown in Figure 5 using frequency related model. The ground wire of the tower and earth resistance are both considered in this kind of model, so the simulation result has a high credibility.

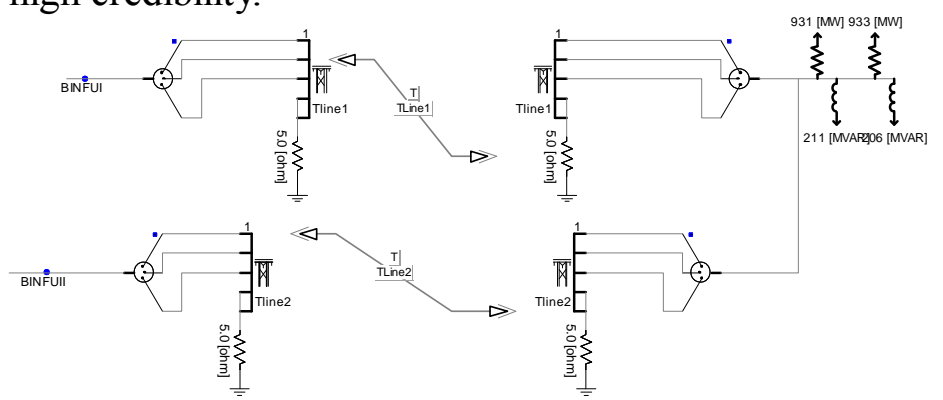

Figure 5 Simulation model of Binfu line based on PSCAD

Three kinds of different transmission line arrangement were simulated and the results are shown in Figure 6 and the effective value of current in each transmission line is shown in Table.4. 


\begin{tabular}{|c|c|c|c|c|c|c|}
\hline \multirow{2}{*}{ Line arrangement } & \multicolumn{3}{|c|}{ Binfu I line } & \multicolumn{3}{c|}{ Binfu II line } \\
\cline { 2 - 7 } & $\mathrm{A}$ & $\mathrm{B}$ & $\mathrm{C}$ & $\mathrm{A}$ & $\mathrm{B}$ & $\mathrm{C}$ \\
\hline Current phase sequence & 1.34 & 1.22 & 1.42 & 1.31 & 1.42 & 1.22 \\
\hline Positive phase sequence & 1.32 & 1.32 & 1.33 & 1.30 & 1.31 & 1.31 \\
\hline Invers phase sequence & 1.32 & 1.32 & 1.33 & 1.30 & 1.31 & 1.31 \\
\hline
\end{tabular}
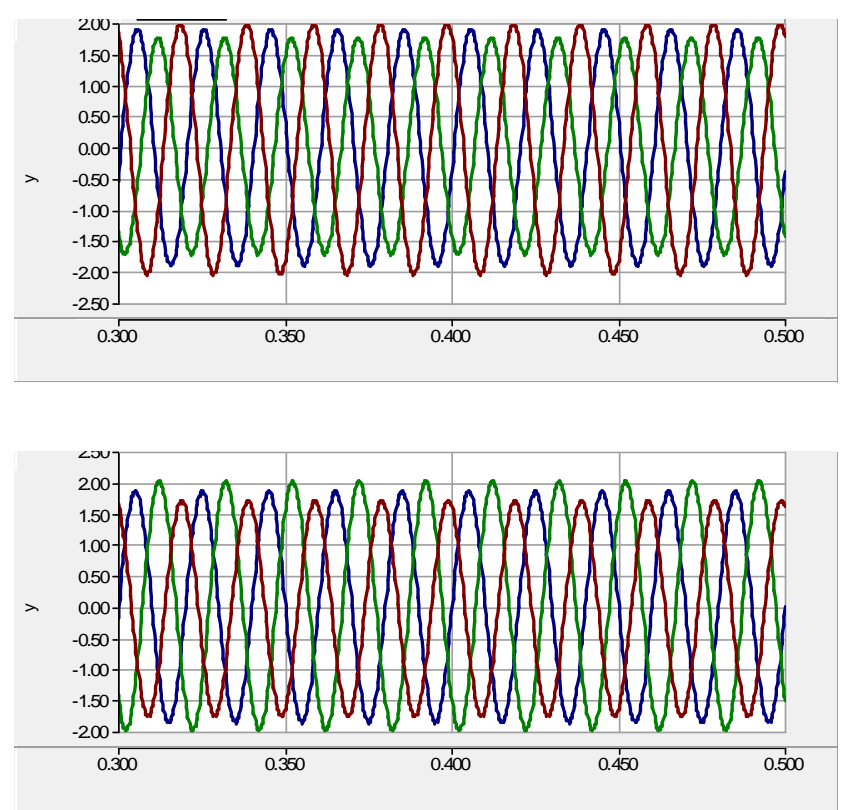

(a) Current arrangement
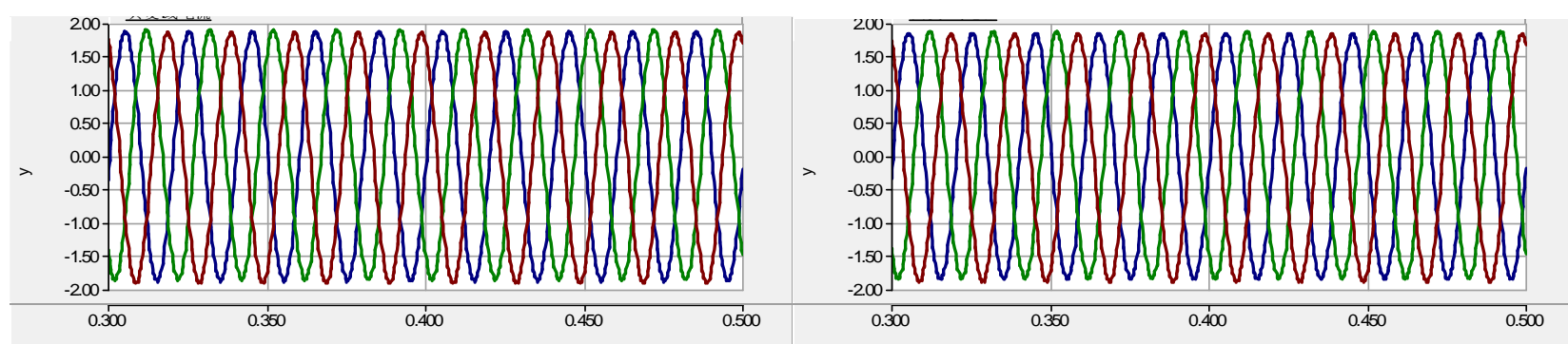

(b) Positive phase sequence arrangement
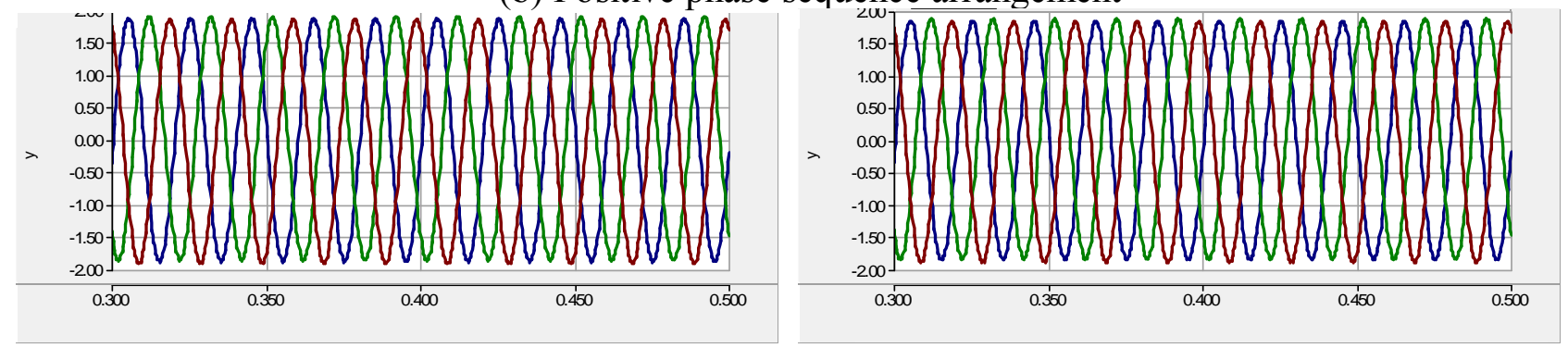

(c) Inverse phase sequence arrangement

Figure 6 Current waveforms of Binfu lines under different arrangement

From Figure 6, it can be seen obviously that unbalanced transmission line parameters is the main reason of the unbalanced phase current. Rearrange the Binfu I line and Binfu II line in inverse sequence can solve this unbalanced current.

\section{Conclusion}

According to related regulations, $500 \mathrm{kV}$ AC overhead transmission line can be nontransposition within $100 \mathrm{~km}$. The unbalance phase current in Binfu lines between Fulong converter 
station and Yibin converter station is induced by non-transposition and unreasonable phase arrangement.

This paper researched the mechanism of unbalance phase current, theoretical analysis and simulation result show that the transmission line arrangement with inverse phase sequence has minimum unbalance. Simulations in PSCAD confirmed this conclusion.

\section{Reference}

[1] Zou lin, Lin fuchang, etc. 2008. Analysis of unbalance factors of transmission lines. Power System Technology. (32):283-286.

[2] Wei gang, Zhang ziyang, Fang zhengliang. 2004. Unbalance analysis on multi transmission lines on the same tower. High Voltage Engineering. (10):9-11.

[3] Wang jiancheng, Zhang tao, Wang qiwen, etc. 2005. Measurement and analysis on unbalance phase current of $500 \mathrm{kV}$ AC transmission line of Qinshan second nuclear power plant. Power System Technology. 29(5):76-80.

[4] Gross, E. T. B. ,and Hesse M H. 1995. Electromagnetic un-transposed lines IEEE, (3):13231336

[5] Hesse M H. 1996. Circulating currents in parallel un-transposed multi circuit line. II. Methods for estimating current unbalanced. IEEE Trans on PSA, 85(3):812-820

[6] GB 50545-2010. Design regulations for $110 \mathrm{kV} \sim 750 \mathrm{kV}$ overhead transmission lines. 